\title{
MODERN METHODS OF ANALYSIS IN POTENTIAL THEORY*
}

\author{
BY G. C. EVANS
}

I shall take the liberty of interpreting the title in terms of a short discussion of certain problems that have been of recent interest, particularly to younger mathematicians, namely, the discontinuous boundary value problems of the Dirichlet and Neumann types, the mixed problem, and finally the generalizations of the potential integral itself to others in which the integrand $1 / r$ is replaced by $1 / r^{\alpha}, 0<\alpha<3$. In this way my review will serve as a continuation of that of the late Professor Kellogg, but will diverge from it in the sort of problem to be considered. $\dagger$

\section{Dirichlet and Neumann Problems}

1. Plane Regions. It is a natural generalization of Poisson's integral to write it in the form of a Stieltjes integral,

$$
u(r, \theta)=u(M)=\frac{1}{2 \pi} \int_{0}^{2 \pi} \frac{a^{2}-r^{2}}{a^{2}+r^{2}-2 a r \cos (\phi-\theta)} d F(\phi),
$$

where $F(\phi)$ is periodic and of bounded variation. If the $d F(\phi)$ is replaced by $d f(e)$ or $f(d e)$ the integral is interpreted as integration with respect to an additive function of sets on the circumference, or in other words, with respect to an arbitrary distribution of positive and negative mass on the circumference, finite in total absolute amount.

The integrand $\left(a^{2}-r^{2}\right) /\left[2 \pi\left(a^{2}+r^{2}-2 a r \cos (\phi-\theta)\right)\right]$ may also be written in terms of the Green's functions $g(M, P)$ or its conjugate $h(M, P)$, in which $P$ is the point on which is performed the integration. In fact, (1) may be written in the form

$$
u(M)=\int_{C} \frac{d h(M, P)}{d \dot{h}(O, P)} d f\left(e_{P}\right)
$$

* An address delivered by invitation of the Program Committee, at the Cambridge Meeting of the Society, September 5, 1936.

$\dagger$ O. D. Kellogg, Recent progress with the Dirichlet problem, this Bulletin, vol. 32 (1926), pp. 601-625. 
in which a factor $a$ has been absorbed in the $f(e)$, and, for a smooth curve,

$$
\frac{d g(M, P)}{d n_{P}} / \frac{d g(O, P)}{d n_{P}}=\frac{d h(M, P)}{d s_{P}} / \frac{d h(O, P)}{d s_{P}}=\frac{d h(M, P)}{d h(O, P)} .
$$

In this form the expression (2) is evidently invariant under conformal transformation, and, if the boundary is regarded as constituted of "prime ends" as elements, ordered according to the values of $h(O, P)$, may be applied to any simply connected region and its boundary (provided that the latter does not consist of a single point). But it is easily seen that a necessary and sufficient condition for (1) is that $u(M)$ be the difference of two functions harmonic and not negative in the circle; hence the same class of functions with reference to the general domain is represented by $(2)$. $^{*}$

But also, except for an additive constant in the integrand, the form (1) may be rewritten in terms of the familiar expression for the potential of a double layer on the circumference. Such an expression, involving a direction of doublets, demands however a certain smoothness in the curve itself, and therefore cannot be extended to general boundaries. Nevertheless, for regions bounded by curves which are sufficiently smooth, it represents again the same class of functions.

Curves of particular importance with respect to such problems are those "of bounded turning," introduced by Radon. $\dagger$ These are curves which have a forward and backward tangent at each point, such that the total variation of the angle turned through by a tangent remains bounded as the whole curve is traced. These curves provide a natural basis for the treatment, in terms of an extension of the theory of integral equations, of mass distributions on them and the related Dirichlet and Neumann problems, and thus generalize to a natural limit the earlier treatment of Plemelj in terms of general mass distribu-

* G. C. Evans, The Logarithmic Potential, American Mathematical Society Colloquium Publications, vol. 6, New York, 1927.

$\dagger \mathrm{J}$. Radon, Über die Randwertaufgaben beim logarithmischen Potential, Sitzungsberichte der Akademie der Wissenschaften in Wien, vol. 128 (1919), pp. 1123-1167. 
tions.* The class of harmonic functions which is typical is rather the class of bounded than merely positive ones. But the analysis involves a much more searching study of linear functional operations than the treatment of continuous density distributions in terms of the Fredholm theory of integral equations, and thus seems, with the work of Fréchet and F. Riesz, to be the basis of much of the modern theory of linear operations. As for the corresponding problem in space, no one seems to have found the proper generalization of curves of bounded turning, the obvious one in terms of the Gaussian sphere not being adequate.

2. Regions in Space with Smooth Boundaries. In three dimensions regions convenient for this sort of study are those with a bounding surface which has a tangent plane at every point and is such that the angle between its normals at nearby points $P, Q$ is dominated by an expression $N \cdot \overline{P Q}$, where $N$ is independent of $P$ and $Q$. The potential of a double layer has the form

$$
U(M)=\int_{s} \frac{\cos \left(r, n_{P}\right)}{r^{2}} d \nu\left(e_{P}\right), \quad r=\overline{M P},
$$

and represents again the class of functions which are differences of two positive harmonic functions. These are given also by a formula analogous to (2). In fact, the connection between $\nu(e)$ and $f(e)$ is stated in terms of Stieltjes integral equations. Moreover the class of functions furnishes unique solutions of the boundary value problems in which is given on $s$ an additive function which is the limit of the integral of $U(M)$ over arbitrary pieces of smooth surfaces approximating to $s$. The function $U(M)$ itself approaches a limiting value as $M$ tends to a point $Q$ of $s$, for almost all $Q$, if $M$ remains within a cone with vertex $Q$ and axis the normal at $Q$, the cone lying within the region. Analogous considerations apply to the Neumann problem and potentials of a single layer. The data given in the boundary problem are the limiting values of the flux, or integral of the normal derivatives, over arbitrary pieces of the approximating surfaces. $\dagger$

* Plemelj, Potentialtheoretische Untersuchen, Leipzig, 1911.

$\dagger$ G. A. Garrett, Necessary and sufficient conditions for potentials of single and double layers; American Journal of Mathematics, vol. 58 (1936), pp. 95129. Reference is given in this memoir to work of H. E. Bray, E. R. C. Miles, C. de la Vallee Poussin, and myself. Some of this same circle of ideas has been 
3. Boundaries Consisting of Regular Points. The form (2), as we have said, we may expect to be carried much further in the direction of general boundaries than the form (3). In two dimensions there was no restriction on the (simply or finitely connected) domain. In three dimensions the question remains as to how far may be carried the representation of functions which are the differences of not negative functions harmonic in the domain-or more simply of functions positive and harmonic in the domain.

A recent statement of this problem is given by Maria and Martin,* direct in the sense that it does not depend on conformal mapping in its analysis. However, it involves the mass function obtained by the sweeping out of unit mass, which is equivalent to the limit on the boundary of the indefinite integral of the normal derivative of the Green's function, and thus in two dimensions to the function $h(M, P)$. What seems essentially novel in three dimensions is the use of the derivative of one positive function of point sets with respect to another, $\dagger$ which yields the analog of the expression $d h(M, P) / d h(O, P)$.

Let then $m(e, M)$ denote the mass distribution obtained by sweeping out unit mass located at a point $M$ of the domain $S$ onto the boundary $s$ of the domain. This is the function whose potential is the solution of the continuous Dirichlet problem corresponding to boundary values $1 / r$ (where $r=M Q, Q$ a boundary point); the distribution function is for given $e$ itself harmonic in $M$, and provides the formula

$$
u(M)=\int_{s} u(P) d m\left(e_{P}, M\right)
$$

for the solution of the Dirichlet problem. $\ddagger$ What turns out to

developed by G. Gunther, Sur une application des intégrales de Stieltjes au problème de Neumann, Comptes Rendus de l'Académie des Sciences, vol. 189 (1929), pp. 447-450; Sur les Intégrales de Stieltjes et leurs Applications, Leningrad, 1932.

* A. J. Maria and R. S. Martin, Representation of positive harmonic functions, Duke Mathematical Journal, vol. 2 (1936), pp. 517-529. 448.

$\dagger$ P. J. Daniell, Stieltjes derivatives, this Bulletin, vol. 26 (1920), pp. 444-

‡C. de la Vallée Poussin, Extension de la méthode du balayage de Poincaré, et problème de Dirichlet, Annales de l'Institut Henri Poincaré, vol. 2 (1932), pp. 169-232. 
be the representation formula for the class of positive harmonic functions is the following:

$$
u(M)=\int_{s} f(P, M) d \mu\left(e_{P}\right),
$$

where $\mu(e)$ is a distribution of positive mass on $s$, and where the function

$$
f(P, M)=\frac{d m(e, M)}{d m\left(e, M_{0}\right)}
$$

is the above mentioned set function derivative taken at the point $P$ on $s$. With the $f(P, M)$ thus fixed, with $M_{0}$ also chosen, to each $u(P)$ there corresponds a $\mu(e)$ and vice versa.

This representation is contingent on the character of the domain and its boundary. The domain need not be simply connected. Every boundary point is assumed to be regular with respect to the Dirichlet problem. It is also assumed that the "principle of Picard" is valid for every point of $s$, that is, a function positive and harmonic in $S$ which vanishes continuously at every point of the boundary $s$ except at one point $Q$ is determined except for a multiplicative constant.

In order to illustrate a situation where this principle is not valid, consider a plane domain $T$ consisting of a circle cut along one radius, and let $Q$ be an interior point of this radius. If $M$ is a point of $T$ there will be two values of $d g(M, Q) / d n$, say $g_{1}(M, Q)$ and $g_{2}(M, Q)$, according as the derivative refers to one side of the cut or the other. The two harmonic functions $g_{1}(M, Q)$ and $g_{2}(M, Q)$ are not negative and vanish as $M$ tends towards the boundary, except at $Q$, and each vanishes for different modes of approach to $Q$. Hence they cannot be linearly dependent.*

Now let $E(u, S)$, with $u \geqq 0$ in $S$ and harmonic, be the set of points $Q$ of $s$ where $\lim \sup u(M)>0$ as $M$ tends to $Q$. Let $u_{n}$

* In two dimensions the order of multiple boundary points is treated by C. Caratheodory with the invention of prime ends. The corresponding Dirichlet problem is discussed in G. C. Evans, The Logarithmic Potential, loc. cit., p. 82.

A significant generalization to three dimensions has been developed by $\mathrm{F}$. W. Perkins, The Dirichlet problem for domains with multiple boundary points, Transactions of this Society, vol. 58 (1925). 
be a sequence of functions of the above kind with limit function $u$. The third requirement on the boundary is that if $E\left(u_{n}, S\right)$ is contained in a closed set $B$ of $s$ for all $n$, the same property holds for $u$.

Finally, a fourth requirement is imposed. For the given domain $S$ there must exist a sequence of nested domains $S_{n}$, satisfying the first three requirements, with the following property. If $M_{0}$ is a point of $S$, and $B$ a closed set in $S+s$, and if $u(M)$ is less than some fixed bound at $M_{0}$, has as domain some $S_{n}$, and is such that $E\left(u, S_{n}\right)$ is contained in $B$, then $u$ is bounded in a neighborhood of any point not in $B$, uniformly with respect to $n$.

These four conditions guarantee the possibility and uniqueness of the representation (4). On the other hand, if the representation is unique, and in all cases $u(M)$ takes on the value zero at any point of $s$ which does not lie on the nucleus of $\mu$ (that is, at a point which can be surrounded by a sphere which contains no mass), the first three conditions follow. In particular, in the plane, it can be shown (by means of conformal transformations) that these four conditions are satisfied, for instance, by the domains bounded by simple closed curves.

4. Poisson's Equation. A positive harmonic function is not always expressible as a Newtonian potential of a distribution of positive mass, since even in the unit sphere a potential of a double layer is required. But a function which is harmonic in the whole of space except on a set where it becomes positively infinite is necessarily the Newtonian potential of a positive mass distributed on a set of capacity zero. Similarly a necessary and sufficient condition that a function, harmonic in a domain, be the potential of a distribution of positive mass on the boundary is evidently given immediately in terms of the Poincare sweeping-out process.

Related to such notions is the question of boundary value problems for Poisson's equation. But for the same degree of generality with respect to mass distributions, the Laplacian

$$
\left(\frac{\partial^{2}}{\partial x^{2}}+\frac{\partial^{2}}{\partial y^{2}}+\frac{\partial^{2}}{\partial z^{2}}\right) u
$$

must be considered as some sort of differential operator as a 
whole. Zaremba's definition, ${ }^{*}$ where $\nabla^{2}(u)$ stands for

$$
\begin{aligned}
u(x+h, y, z) & +u(x-h, y, z)+u(x, y+h, z)+\cdots \\
& +u(x, y, z-h)-6 u(x, y, z),
\end{aligned}
$$

allows one to treat Poisson's equation $\nabla^{2} u=-4 \pi f(x, y, z)$ without imposing a Hölder condition on the density $f(x, y, z)$ of the mass distribution. Still more general is the expression of Poisson's equation in the form

$$
\int_{s} \frac{d u}{d n} d s=4 \pi m(s),
$$

where $m(s)$ is the regularized function of surfaces corresponding to an arbitrary distribution of mass $m(e) . \dagger$

\section{The Mixed Problem}

5. A Uniqueness Theorem. The difficulty in the mixed problem, in which limiting values which the harmonic function itself takes on are given on a part of the boundary, and the values which the limit of the normal derivative (or indefinite integral of the normal derivative takes on) are given on the rest, lies in the specification of a convenient class of functions in which the solutions are unique. This difficulty seems to underlie also the application of the method of integral equations to the problem, for these carry with them some implication of function class. Accordingly we content ourselves with the working out of a fairly simple example of the problem, merely citing some of the bibliography of other studies. $\ddagger$ The solution will be given in terms of the simpler Dirichlet problem.

* S. Zaremba, Contribution à la théorie d'une équation fonctionelle de la physique, Rendiconti del Circolo Matematico di Palermo, vol. 19 (1905), pp. $140-150$.

H. Petrini, Les dérivées premières et secondes du potentiel, Acta Mathematica, vol. 31 (1908), pp. 127-332.

$\dagger$ G. C. Evans, Discontinuous boundary value problems of the first kind for Poisson's equation, American Journal of Mathematics, vol. 51 (1929), pp. 1-18.

$\ddagger \mathrm{S}$. Zaremba, Sur un problème mixte relatif a l'équation de Laplace, Bulletin de l'Académie des Sciences de Cracow, 1910, pp. 313-344.

L. Lichtenstein, Randwertaufgaben der linearen elliptischen Gleichungen, Journal für die reine und angewandte Mathematik, vol. 143 (1913); see pp. 93105.

H. Villat, Sur la résolution de certaines équations intégrales, et sur quelques problèmes qui s'y rattachent, Acta Mathematica, vol. 40 (1916); see pp. 162-167. 
Let $S$ be an open circular region, $s$ the circumference, $O$ the center, and $F$ a closed set of points on $s$ of positive capacity.* Denote by $s-F$ the sum of arc-intervals $I_{1}, I_{2}, \cdots$, which is complementary to $F$ with respect to $s$, and by $C F$ the complement of $F$ with respect to the whole plane. Let $f(P)$ be a function which is defined and continuous on $F$, and $h(e)$ a completely additive function of sets on $s-F$, that is, completely additive on each interval $I_{k}$ and such that its total variation is bounded on $\sum I_{k}$.

We note first the following uniqueness theorem:

THEOREM. There cannot be more than one function $u(M)$ which is harmonic in $S$ and

(a) is bounded in a neighborhood of $F$;

$\left(\mathrm{a}^{\prime}\right)$ takes on the values $f(P)$ as $M$ in $S$ tends to $P$ in $F$, except for a subset of points $P$ of zero capacity;

(b) is such that $\int_{0}^{A}(d u / d n) d s$ is bounded as $A$ in $S$ tends to $P$ in any closed subinterval of each $I_{k}$;

$\left(\mathrm{b}^{\prime}\right)$ is such that $\int_{A}^{B}(d u / d n) d s$ tends to $h(P Q)$ where $P Q$ is an arc interval and $A$ tends to $P, B$ to $Q$ in an interval $I_{k}$ of $s-F$, except for a denumerable infinity of points $P, Q$.

In fact, if $w(M)$ is the difference of two possible $u(M)$, and $v(M)=\int_{0}^{M}(d w / d n) d s$ its conjugate function, the function $v(M)$ is bounded as $M$ tends to the boundary in any closed interval contained in an $I_{k}$ and takes on a constant value $c_{k}$ except at a denumerable infinity of points $P$ of $I_{k}$. It is therefore harmonic except for removable discontinuities at every point of $I_{k}$ and uniquely extensible across it, by means of the formula

$$
v(M)-c_{k}=c_{k}-v\left(M^{\prime}\right), \quad M^{\prime} \text { inverse to } M \text { in } s .
$$

It follows that $w(M)$ can be made harmonic at every point of $I_{k}$ and that $w(M)$ is extensible across the cut $I_{k}$ by means of a formula

G. C. Evans and R. N. Haskell, The mixed problem for Laplace's equation in the plane. Discontinuous boundary values, Proceedings of the National Academy, vol. 16 (1930), pp. 620-526.

H. Hornich, Lösung einer vermischten Randwertaufgabe der Potentialtheorie durch hyperelliptische Integrale, Monatshefte für Mathematik und Physik, vol. 39 (1932), pp. 107-128.

* A bounded set is of zero capacity if it will bear no positive mass distribution with a bounded potential. It is of positive capacity if it will bear a positive mass distribution with a bounded potential; see de la Vallée Poussin, loc. cit. 


$$
w\left(M^{\prime}\right)-w(M)=\gamma_{k}, \quad \gamma_{k} \text { a constant. }
$$

But since $w(M)$ is harmonic on $I_{k}$, we have

$$
w\left(M^{\prime}\right)=w(M),
$$

and the function so defined is single valued and harmonic in the whole plane except on $F$, and bounded. It follows then that if $w(M)$ is not identically 0 , the set of points $P$ on $F$ where $\lim \sup _{M=P} w(M)>0$ or $\lim \inf _{M=P} w(M)<0$ contains a closed set of positive capacity, as $M$ in $C F$ tends to $P$. $^{*}$

This statement, however, contradicts our hypothesis. In fact, let $Q$ be a point of $F$ not in the exceptional set for $w(M)$ as defined in the hypothesis $\left(a^{\prime}\right)$ of the theorem. Let $M_{1}, M_{2}, \cdots$, $M_{n}, \cdots$ be a sequence of points in $C F$ tending to $Q$. To these there corresponds a sequence $N_{n}$ in $S$, tending to $Q$, where $N_{n}=M_{n}$ if $M_{n}$ is in $S$,

$N_{n}=M_{n}^{\prime}$ if $M_{n}$ is in $C(S+s)$,

$N_{n}$ lies in $S$ sufficiently close to $M_{n}$, if $M_{n}$ lies on an interval $I_{k}$.

The $N_{n}$ may therefore be chosen so that $0=\lim _{n=\infty} u\left(N_{n}\right)$ $=\lim _{n=\infty} u\left(M_{n}\right)$. Hence, for $M$ in $C F, \lim _{M=Q} w(M)=0$ except for $Q$ in a subset of $F$ of zero capacity.

We conclude then that $w(M) \equiv 0$ and $u(M)$ is unique.

6. Construction of a Solution. Let $u(M)$ be the solution of the Dirichlet problem for the infinite region of boundary $F$ corresponding to the given values $f(P)$. Such a solution exists, and takes on the boundary values $f(P)$ continuously, except at the irregular points of $F$. These in fact constitute a subset (not necessarily closed) of zero capacity.

The boundary values are taken on as $M$ tends to $P$ from $C F$, and therefore, in particular, from $S$. Moreover we note that $u_{1}(M)=u_{1}\left(M^{\prime}\right)$, since the problem is unchanged by inversion with respect to the circumference $s$.

In particular, let $g_{F}(A, B)$ be the Green's function for the domain $C F$. Then again, with consideration of the inversion,

$$
g_{F}(A, B)=g_{F}\left(A^{\prime}, B^{\prime}\right) .
$$

We shall consider briefly the properties of the function

$$
u_{2}(M)=-\frac{1}{\pi} \int_{s-F} g_{F}(M, P) d h\left(e_{P}\right)=u_{2}\left(M^{\prime}\right) .
$$

* O. D. Kellogg, Foundations of Potential Theory, Berlin, 1929; see p. 335. 
Let $A, B$ be two points of $S$, which we shall allow ultimately to tend to two points $X, Y$ respectively of the same interval $I_{k}$ of $s-F$, which are points of continuity of $h(e)$, the polar angle of $X$ being less than that of $Y$; and let $l$ be a curve joining $A, B$, with a continuously turning tangent. We shall choose as a direction of the normal $n$ at a point of $l$ that direction which points toward the center when $l$ has been deformed into $X Y$, and define $d s=|d s|$. When $A$ and $B$ have become near to $X, Y$ the flux

$$
N=\int_{A}^{B} \frac{d u}{d n} d s=-\frac{1}{\pi} \int_{s-F} d h\left(e_{P}\right) \int_{A}^{B} \frac{d g_{F}}{d n_{M}} d s
$$

may be evaluated in simple terms.

In fact, if we make an inversion in the circle $s$, letting $n^{\prime}$ be the direction corresponding to $n$ by the inversion, we have

$$
\begin{aligned}
2 N= & \int_{A}^{B} \frac{d u}{d n} d s+\int_{A^{\prime}}^{B^{\prime}} \frac{d u}{d n^{\prime}} d s^{\prime}=\int_{A}^{B} \frac{d u}{d n} d s+\int_{B^{\prime}}^{A^{\prime}} \frac{d u}{d n^{\prime}} d s^{\prime}, \\
-2 N= & \frac{1}{\pi} \int_{s-F} d h\left(e_{P}\right)\left\{\int_{A}^{B}\left[\frac{d}{d n} \log \frac{1}{M P}+\frac{d}{d n} \gamma(M, P)\right] d s\right. \\
& \left.+\int_{B^{\prime}}^{A^{\prime}}\left[\frac{d}{d n^{\prime}} \log \frac{1}{M^{\prime} P}+\frac{d}{d n^{\prime}} \gamma\left(M^{\prime}, P\right)\right] d s^{\prime}\right\},
\end{aligned}
$$

where $\gamma(M, P)$ is harmonic and bounded, with its derivatives, as long as $M$ is at a distance from $F$ which is at least as great as some constant $\delta>0$. We can therefore take $A$ and $B$ near enough to $X$ and $Y$ so that the integrals of $\left|d \gamma(M, P) / d n_{M}\right|$ along radii from $A$ to $A^{\prime}$ and $B$ to $B^{\prime}$ will be as small as we please. Hence

$$
\begin{aligned}
-2 N= & \frac{1}{\pi} \int_{s-F} d h\left(e_{P}\right)\left\{\int_{A}^{B} \frac{d}{d n} \log \frac{1}{M P} d s\right. \\
& \left.+\int_{B^{\prime}}^{A^{\prime}} \frac{d}{d n^{\prime}} \log \frac{1}{M^{\prime} P} d s^{\prime}\right\}+\eta(A, B),
\end{aligned}
$$

where given $\epsilon>0$, we can take $A, B$ close enough to $X, Y$ so that

$$
|\eta(A, B)|<\epsilon .
$$

In fact the integral of the exterior normal derivative of $\gamma(M, P)$ around the closed contour $A B B^{\prime} A^{\prime} A$ is zero. Moreover 


$$
\begin{aligned}
& \int_{A}^{B} \frac{d}{d n} \log \frac{1}{M P} d s=-\Varangle B P A, \\
& \int_{B^{\prime}}^{A^{\prime}} \frac{d}{d n^{\prime}} \log \frac{1}{M^{\prime} P} d s^{\prime}=-\Varangle A^{\prime} P B^{\prime}
\end{aligned}
$$

by direct computation.

Accordingly

$$
\lim _{\substack{A=X \\ B=Y}} N=\frac{1}{2 \pi} \lim _{\substack{A=X \\ B=Y}} \int_{s-F}\left(\Varangle B P A+\Varangle A^{\prime} P B^{\prime}\right) d h\left(e_{P}\right)
$$

and since the integrand remains bounded as $A$ tends to $X$ and $B$ to $Y$, and $X, Y$ are not point masses of $h(e)$, it follows that

$$
\lim _{\substack{A=X \\ B=Y}} N=h\left(I_{X Y}\right),
$$

where $I_{X Y}$ stands for the arc interval $X, Y$. There are, of course, at most a denumerable infinity of point masses of $h(e)$.

The function

$$
u(M)=u_{1}(M)+u_{2}(M)
$$

is a type of solution of the mixed boundary value problem. In order to bring it into the class of solutions to which the uniqueness theorem applies, we must, however, make restrictions on the set function $h(e)$ or the set $F$, or both. The function $u_{1}(M)$ gives no trouble. The function $u_{2}(M)$, if $h(e)$ is not restricted, may however become infinite at points which are ends of intervals $I_{k}$, and even at certain other points-if for example $F$ is a perfect non-dense set on $s$. The points of $F$ which are limit points of $s-F$ need not constitute a set of zero capacity.

On the other hand, if $h(e)$ is absolutely continuous and its derivative is bounded, the function $u_{2}(M)$ vanishes continuously at every regular point of $F$; for $g_{F}(M, P)$ is the Green's function for $F$, and it vanishes at regular points of $F$, and, since $0 \leqq g_{F}(M, P) \leqq \log 1 / \overline{M P}$, the absolute continuity of $\int_{s-F} g_{F}(M, P) h^{\prime}(P) d s_{P}$ is uniform as $M$ approaches a point $Q$ which is in $F$.

THEOREM. If $h(e)$ is absolutely continuous, with a bounded derivative, the function $u(M)=u_{1}(M)+u_{2}(M)$, where $u_{1}(M)$ and $u_{2}(M)$ are the functions defined in this section, is a solution of the mixed boundary value problem and belongs to the class of functions to which the uniqueness theorem of $\$ 5$ applies. 
Another special case arises if $F$ consists merely of a finite number of closed intervals, and $h(e)$ is absolutely continuous with a bounded derivative in a neighborhood of the end points of each interval of $s-F$, so that $u_{2}(M)$ remains bounded in a neighborhood of $F$.

Hornich considers this latter case, with continuously given boundary data, and also generalizes the problem by considering the case in which along the real axis values of the quantity

$$
\frac{\partial u}{\partial y}+\lambda_{i} \frac{\partial u}{\partial x}=h
$$

are assigned on certain intervals ( $b$ intervals) of the boundary, while the values of $u$ itself are assigned on other intervals ( $a$ intervals). ${ }^{*}$ His method is likewise an extension of his method used in the earlier problem, namely, the construction of a Green's function as the real part of a function of a complex variable, with branch points at the ends of the intervals. In this case, however, the function is not uniquely determined, if it exists, unless the $a$ - and $b$-intervals alternate.

A related problem is the three dimensional one, discussed by Bouligand, Giraud, and Delens, $\uparrow$ where the boundary values of a slanting derivative are given. If the direction of the given derivative is nowhere tangent to the boundary, a uniqueness theorem can be deduced inmediately. For the difference of two such solutions would be one with a zero slanting derivative, and, for sufficiently smooth boundaries, if this solution were not identically zero, the derivative could not be zero at the points of the boundary at which the function took on its highest and lowest bounds. By construction of a generalized Green's function (of which the Laplacian is not zero), based on a "potential of an oblique double layer," the solution may be written explicitly, provided that the given derivative is subject to a certain integral condition of compatibility. The differential geometry of the situation in the case where the homogeneous problem

* H. Hornich, Die allgemeine vermischte Randwertaufgabe der ebenen Potentialtheorie, Monatshefte für Mathematik und Physik, vol. 39 (1932), pp. 455-460, and vol. 41 (1934), pp. 7-19.

† G. Bouligand, G. Giraud et P. Delens, Le Problème de la Dérivée Oblique en Théorie du Potentiel, Paris, 1935. 
has solutions, the given direction being somewhere tangent to the boundary, may be developed in terms of a special theory of congruences.

\section{iII. Potentials in Space, not Newtonian}

7. Form of Integral. The potentials to be considered are of the form

$$
u(M)=\int_{F} \frac{1}{r^{\alpha}} d m\left(e_{P}\right), \quad r=\overline{M P}, \quad 0<\alpha<3,
$$

in which the mass is located on a closed bounded set $F$ in space. These do not seem to be subject to differential equations-excluding fractional derivatives-unless the mass is localized on a set of special character. For instance, if the mass lies in the plane $x=0$, the potential for values of $x \neq 0$ satisfies the equation

$$
\nabla^{2} u+\frac{4 \alpha-1}{x} \frac{\partial u}{\partial x}=0,
$$

which is called Green's equation by Pierre Humbert.* The same remark applies to potentials of more general form where $1 / r^{\alpha}$ is replaced by a suitable function $\Phi(r)$.

Of special interest, however, are the results which are independent of particular forms of mass distribution, and thus do not depend on the methods of differential equations. In this direction, the recent thesis of Otto Frostman $\dagger$ deals with the problem of capacity and conductor potential, as related to the transfinite diameter of Fekete and Pólya and Szeg̈̈, $\ddagger$ and makes application to the complex variable on the two dimensional sphere. The present section may be regarded as an introduction to the work of Frostman. The problem which we take up will be that of the conductor potential. Our methods will differ only slightly from those of Frostman and we shall only slightly extend his results.

* Humbert, Potentiels et Prépotentiels, Paris, 1936.

$\dagger$ O. Frostman, Potentiel d'Équilibre et Capacitê des Ensembles, Lund, 1935.

¥ Pólya and Szegö, Transfiniter Durchmesser ebener und räumlicher Punktmengen, Journal für die reine und angewandte Mathematik, vol. 165 (1931), pp. 4-49. 
8. Minimum Energy. The intrinsic energy, or simply the energy of a distribution, is defined as the iterated integral

$$
I=\int_{F} \int_{F} \frac{1}{\overline{M P}^{\alpha}} d \sigma\left(e_{P}\right) d \sigma\left(e_{M}\right) .
$$

It is a quantity which Frostman shows to be positive, if finite, when $\sigma(e)$ is any additive function of point sets, not necessarily positive; in fact $I=0$ only if $\sigma(e) \equiv 0$. This result is obtained by rewriting $I$ as the integral over space of a square of a potential, making use of the identity

$$
\int_{W} \frac{1}{M P^{\beta}} \frac{1}{P Q^{\gamma}} d P=\text { const. } \frac{1}{M Q^{\alpha}}, \quad\left\{\begin{array}{c}
\alpha=\beta+\gamma-3 \\
W=\text { entire space }
\end{array}\right.
$$

and taking $\beta=\gamma$. An equilibrium distribution or conductor distribution, if it exists, is a distribution of a given total mass on $F$ in such a way as to give to $I$ its minimum value; the resulting potential is the equilibrium or conductor potential.

For convenience in this exposition it will be said that a set $F$ is of zero capacity if the energy of any distribution of positive mass located on it is $+\infty$, and of positive capacity if it is possible to distribute positive mass on it in such a way that $I$ remains finite. ${ }^{*}$ If $F$ is of positive capacity and $\mu(e)$ and $\lambda(e)$ are two distributions of positive mass on $F$ such that the energies $I(\mu)$ and $I(\lambda)$ are finite, it can be seen that $\mu(e)+\lambda(e)$ is another such distribution, and also that the quantity

$$
I(\mu, \lambda)=\int_{F} \frac{1}{\overline{M P}^{\alpha}} d \mu\left(e_{M}\right) d \lambda\left(e_{P}\right)
$$

is finite. In fact

$$
I(\lambda+\mu)=I(\lambda)+2 I(\lambda, \mu)+I(\mu) .
$$

Suppose then that we have two closed bounded sets of positive capacity, $F_{1}, F_{2}$, with no point in common, and therefore at a positive distance $\delta$, and that a distribution of positive mass $\mu(e)$ of total mass $\mu$ is spread on $F_{1}$ and a distribution of negative mass $-\nu(e)$, of total value $-\nu$, is spread on $F_{2}$. Does there

* It can be shown that this definition is equivalent to that in which the words "its potential remains bounded" are substituted for the words " $I$ remains finite." 
exist such an arrangement for which the energy $I$ takes on a minimum value?

We write

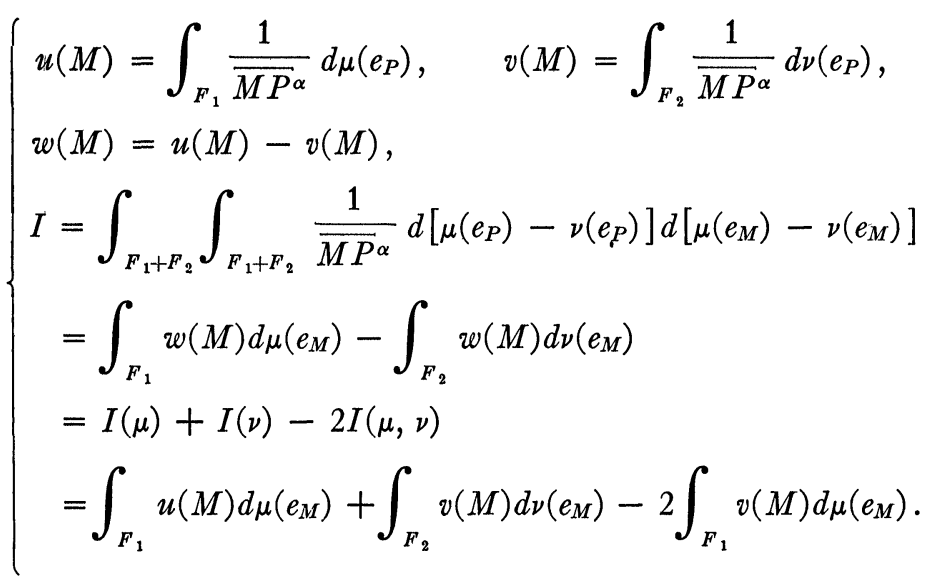

Let $\mu_{n}(e), \nu_{n}(e), n=1,2, \cdots$, be a sequence of pairs of distributions with the given total masses for which the corresponding values $I_{n}$ of $I$ tend to the lower bound $I_{0}$ of $I$. Since $\mu_{n}\left(F_{1}\right)=\mu$, $\nu_{n}\left(F_{2}\right)=\nu$, there exists a subsequence of $\{n\}$ for which these mass distributions converge in the weak sense to mass distributions $\mu_{0}(e), \nu_{0}(e)$ respectively. And without loss of generality we may regard all the values of $n$ as restricted to this subsequence.

Let $I^{\prime}$ denote the energy of the distribution $\mu_{0}(e)-\nu_{0}(e)$ and $u_{0}(M), v_{0}(M)$ the respective potentials of $\mu_{0}(e)$ and $\nu_{0}(e)$. We wish to show that $I^{\prime}=I_{0}$.

The function $1 / \overline{M P}^{\alpha}$ is lower semi-continuous if $M$ and $P$ both lie on $F_{1}$ (or $F_{2}$ ) and is continuous if $M$ lies on $F_{1}$ and $P$ on $F_{2}$. Hence, by the weak convergence, ${ }^{*}$

$$
\begin{gathered}
\liminf _{n=\infty} I\left(\mu_{n}\right) \geqq I\left(\mu_{0}\right), \quad \lim _{n=\infty} \inf I\left(\nu_{n}\right) \geqq I\left(\nu_{0}\right), \\
\lim _{n=\infty} I\left(\mu_{n}, \nu_{n}\right)=I\left(\mu_{0}, \nu_{0}\right),
\end{gathered}
$$

and

$$
I_{0} \geqq I^{\prime}
$$

* As in Frostman, loc. cit., p. 33. 
But $\mu_{0}(e), \nu_{0}(e)$ are admissible distributions, so that

$$
I^{\prime} \geqq I_{0}
$$

It follows that $I^{\prime}=I_{0}$, and the distribution $\sigma_{0}(e)=\mu_{0}(e)-\nu_{0}(e)$ is one for which $I$ takes on its lower bound.

9. The Equilibrium Distribution. It remains to find the character of such a conductor distribution $\sigma_{0}(e)=\mu_{0}(e)-\nu_{0}(e)$. To this end we let $\delta \mu(e)$ be a distribution on $F_{1}$ for which the energy $I(\delta \mu)$ is finite; it follows that $I\left(\mu_{0}+\delta \mu\right)$ and $I\left(\mu_{0}, \delta \mu\right)$ are also finite. By substitution, it may be verified that the change of $I$ is given by the formula

$$
\begin{aligned}
\Delta I= & -2 \int_{F_{2}} \int_{F_{1}} \frac{1}{\overline{Q P}^{\alpha}} d \nu_{0}\left(e_{P}\right) d \delta \mu\left(e_{Q}\right) \\
& +2 \int_{F_{1}} \int_{F_{1}} \frac{1}{\overline{Q^{\alpha}}} d \mu_{0}\left(e_{P}\right) d \delta \mu\left(e_{Q}\right) \\
& +\int_{F_{1} F_{1}} \frac{1}{\overline{Q P}^{\alpha}} d \delta \mu\left(e_{P}\right) d \delta \mu\left(e_{Q}\right) \\
= & 2 \int_{F} w_{0}(Q) d \delta \mu\left(e_{Q}\right)+I(\delta \mu),
\end{aligned}
$$

where

$$
\Delta I \geqq 0 \text { if } \int_{F} d \delta \mu\left(e_{Q}\right)=0 \text { and } \mu_{0}(e)+\delta \mu(e) \geqq 0
$$

The conditions in $\left(11^{\prime}\right)$ can be met if we write (making use of the principle of the Lagrangian multiplier)

$$
\delta \mu(e)=\epsilon\left\{\phi(e)-\frac{\phi\left(F_{1}\right)}{\mu_{0}\left(F_{1}\right)} \mu_{0}(e)\right\}, \quad \epsilon>0,
$$

where $\phi(e)$ is a positive mass distribution on $F_{1}$, and $\epsilon$ is small enough, $\phi(e)$ being chosen so that $I(\phi)$ is finite. Then $I(\delta \mu)$ is also finite, and from $\left(11^{\prime}\right)$ it is deduced as a necessary condition that

$$
\int_{F_{1}} w_{0}(Q) d \delta \mu\left(e_{Q}\right) \geqq 0 ;
$$


for otherwise $\Delta I$ would be $<0$ for $\epsilon$ small enough. We have then the necessary condition

$$
\int_{F_{1}} w_{0}(Q) d\left\{\phi\left(e_{Q}\right)-\frac{\phi\left(F_{1}\right)}{\mu_{0}\left(F_{1}\right)} \mu_{0}\left(e_{Q}\right)\right\} \geqq 0,
$$

which, by defining

$$
J_{1}=\frac{1}{\mu_{0}\left(F_{1}\right)} \int_{F_{1}} w_{0}(Q) d \mu_{0}\left(e_{Q}\right),
$$

may be written in the form

$$
\int_{F_{1}}\left[w_{0}(Q)-J_{1}\right] d \phi\left(e_{Q}\right) \geqq 0,
$$

where $\phi(e) \geqq 0$ and $I(\phi)$ is finite.

It follows that $w_{0}(Q) \geqq J_{1}$ everywhere on $F_{1}$ except possibly on a subset whose capacity in our sense is zero.

Denote now by $F_{1}^{\prime}$ the closed cover of the subset of $F_{1}$ on which the distribution $\mu_{0}(e)$ lies, so that any point in $F_{1}-F_{1}^{\prime}$ may be surrounded by a sphere which contains no mass, and that property holds for no point of $F_{1}^{\prime}$. Then we shall see that at every point of $F_{1}^{\prime}, w_{0}(Q) \leqq J_{1}$.

In fact, if $P$ is a point where $w_{0}(P)>J_{1}+2 \eta, \eta>0$, we can surround $P$ by a sphere in which everywhere $w_{0}(Q) \geqq J_{1}+\eta$; for $w_{0}(Q)$ is lower semi-continuous. By hypothesis, this sphere $\Gamma$ contains a portion of the mass $\mu_{0}(e)$. Take now $\phi(e)=-\mu_{0}(\Gamma \cdot e)$. The conditions in $\left(11^{\prime}\right)$ will still be met if $\epsilon$ is small enough and $\delta \mu_{0}(e)$ is given by $\left(11^{\prime \prime}\right)$. But now, for small enough $\epsilon$ we shall have $\Delta I<0$, since the left hand member of (12) will be negative. This result is in contradiction with $\left(11^{\prime}\right) .{ }^{*}$

A similar result with inequality signs reversed is obtained for $F_{2}$, when we write

$$
J_{2}=\frac{1}{\nu_{0}\left(F_{2}\right)} \int_{F_{2}} w_{0}(Q) d \nu_{0}\left(e_{Q}\right) .
$$

The result to this point may be summarized as follows:

* At this point, our solution answers the problem of the conductor distribution of positive mass on $F_{1}$ of given total amount, under the influence of an arbitrary fixed distribution of mass on $F_{2}$. 
Given a distribution of positive mass on $F_{1}$ of total amount $\mu$ and of negative mass on $F_{2}$ of total amount $-\nu, F_{1}$ and $F_{2}$ being closed bounded sets of positive capacity, with no point in common, there will be distributions $\mu_{0}(e),-\nu_{0}(e)$ which will make the total energy (9) a minimum.

There will be two constants, $J_{1}, J_{2}$ such that the potential w(M) of $\mu_{0}(e)-\nu_{0}(e)$ will, except for possible subsets of capacity zero, satisfy the relations

$$
\begin{aligned}
& w_{0}(M) \geqq J_{1}, \quad M \text { on } F_{1} ; \quad w_{0}(M) \leqq J_{2}, \quad M \text { on } F_{2}, \\
& w_{0}(M)=J_{1}, \quad M \text { on } F_{1}^{\prime} ; \quad w_{0}(M)=J_{2}, \quad M \text { on } F_{2}^{\prime},
\end{aligned}
$$

where $F_{1}^{\prime}$ and $F_{2}^{\prime}$ are the portions of $F_{1}$ and $F_{2}$ respectively which bear the masses $\mu_{0}(e), \nu_{0}(e)$.

The integral $I$ thus has the value

$$
I_{0}=J_{1} \mu-J_{2} \nu \text {. }
$$

We have also the following result:

The minimizing distribution is unique.

Suppose in fact that $m_{0}(e),-n_{0}(e)$ form a second distribution on $F_{1}$ and $F_{2}$ respectively for which the energy is $I_{0}$, the total amounts being $\mu,-\nu$, the same as before. Writing $\sigma(e)=\mu_{0}(e)$ $-\nu_{0}(e), \sigma^{\prime}(e)=m_{0}(e)-n_{0}(e), J_{1}^{\prime}, J_{2}^{\prime}$ as the constant values of the new potential, we have

$$
I\left(\sigma-\sigma^{\prime}\right) \geqq 0 \text {. }
$$

But

$$
\begin{aligned}
I\left(\sigma-\sigma^{\prime}\right) & =I(\sigma)+I\left(\sigma^{\prime}\right)-2 I\left(\sigma, \sigma^{\prime}\right) \\
& =J_{1} \mu-J_{2} \nu+J_{1}^{\prime} \mu-J_{2}^{\prime} \nu-\int w_{0} d \sigma^{\prime}-\int w_{0}^{\prime} d \sigma,
\end{aligned}
$$

and

$$
\begin{aligned}
& \int w_{0} d \sigma^{\prime}=\int_{F_{1}} w_{0} d \mu_{0}^{\prime}-\int_{F_{2}} w_{0} d \nu_{0}^{\prime}=\left(J_{1}+\epsilon_{1}\right) \mu-\left(J_{2}-\epsilon_{2}\right) \nu, \\
& \int w_{0}^{\prime} d \sigma=\int_{F_{1}} w_{0}^{\prime} d \mu_{0}-\int_{F_{2}} w_{0}^{\prime} d \nu_{0}=\left(J_{1}^{\prime}+\epsilon_{3}\right) \mu-\left(J_{2}^{\prime}-\epsilon_{4}\right) \nu,
\end{aligned}
$$


where by the theorem stated above (for example, $w_{0} \geqq J_{1}$ on $F_{1}$ ), we have $\epsilon_{i} \geqq 0, i=1,2,3,4$. Hence,

$$
I\left(\sigma-\sigma^{\prime}\right)=-\epsilon_{1} \mu-\epsilon_{2} \nu-\epsilon_{3} \mu-\epsilon_{4} \nu \leqq 0 .
$$

From this and the previous inequality it follows that $I\left(\sigma-\sigma^{\prime}\right)$ $=0$, and, by Frostman's theorem, that $\sigma(e) \equiv \sigma^{\prime}(e)$ for all sets $e$ measurable $(B)$. But then the potentials of the two mass distributions are also identical.

10. Total Masses Variable. We may consider various values of the total masses $\mu, \nu$. If $\nu=0, \mu=1$ we obtain the "equilibrium distribution" for a unit positive mass on $F_{1}$, as considered especially for $\alpha \geqq 1$ in the memoir cited. We notice however that there is also a unique equilibrium distribution for the case $0<\alpha<1$, under the same conditions. We denote the energy in this case by $i_{1}$.

Let $\mu=1, \nu=-1$ and write $i_{0}=J_{1}-J_{2}$. Let $\sigma(e)=\mu(e)-\nu(e)$, where $\mu(e),-\nu(e)$ are distributions of total amounts $m,-m$ on $F_{1}$ and $F_{2}$ respectively, and define the averages

$$
\bar{w}_{1}=\frac{1}{m} \int_{F_{1}} w(P) d \mu\left(e_{P}\right), \quad \bar{w}_{2}=\frac{1}{m} \int_{F_{2}} w(P) d \nu\left(e_{P}\right) .
$$

If $\mu(e),-\nu(e)$ are not identical with $m \mu_{0}(e),-m \nu_{0}(e)$ respectively on all sets measurable $(B)$, then

$$
I(\sigma)>m^{2} i_{0}>0 ; \quad \bar{w}_{1}-\bar{w}_{2}>m i_{0}>0 .
$$

In fact, $m^{2} i_{0}=I_{0}<I(\sigma)=m\left(\bar{w}_{1}-\bar{w}_{2}\right)$.

Finally, let us take $\mu, \nu$ variable, but $\mu=\nu+1$ so that we shall consider the equilibrium distribution of a total algebraic mass of amount $\mu-\nu=1$, the positive mass $\mu(e)$ on $F_{1}$ and the negative mass $-\nu(e)$ on $F_{2}$. We show that under these conditions, with $\mu, \nu$ variable and $\mu=\nu+1$, there is an optimum distribution which makes I a minimum. Evidently in this case $J_{1}=J_{2}=I_{0}$, the minimum value.

In fact, the reasoning of $\S 8$ of this Part III applies unless for every infinite subsequence of the $\mu_{n}(e), \nu_{n}(e)$ the $\nu_{n}\left(F_{2}\right)$ fail to be bounded. In order to eliminate this possibility, it will be shown that $I$ becomes indefinitely large with $\nu$ and hence cannot thus approach its lower bound. 
Write $\mu(e)=p(e)+m(e)$ with $p(e) \geqq 0, m(e) \geqq 0, p\left(F_{1}\right)=1$, so that $m\left(F_{1}\right)=\nu\left(F_{2}\right)=\nu$. Let the potential of $p(e)$ be $u^{\prime}(M)$ and that of $m(e)-\nu(e)$ be $w^{\prime}(M)$. Then

$$
\begin{aligned}
I= & \int_{F_{1}}\left[u^{\prime}(M)+w^{\prime}(M)\right] d\left[p\left(e_{M}\right)+m\left(e_{M}\right)\right] \\
& -\int_{F_{2}}\left[u^{\prime}(M)+w^{\prime}(M)\right] d \nu\left(e_{M}\right) \\
= & \left\{\int_{F_{1}} w^{\prime} d m-\int_{F_{2}} w^{\prime} d \nu\right\}+\int_{F_{1}} u^{\prime} d p+\int_{F_{1}} u^{\prime} d m-\int_{F_{2}} u^{\prime} d \nu,
\end{aligned}
$$

or

$I \geqq \nu^{2} i_{0}+i_{1}+0-\nu / \delta^{\alpha}$,

$\delta$ being the minimum distance between points of $F_{1}$ and $F_{2}$. Accordingly $I$ becomes infinite with $\nu$.

11. Special Case. Nothing has been said about the more interesting and important problem in which $\delta$ may be zero, or in which $F_{1}$ and $F_{2}$ overlap. It is not certain that this general problem has a solution. The simplest possible problem of this kind is that of two concentric spherical surfaces, where the radius of the inner surface is variable. We shall see that a solution of the problem exists in this case. We take $\alpha<1$.

The formula for the potential of unit mass, spread uniformly on a spherical surface of radius $r$, at a point distant $\xi$ from the center is found by integration to be the following:

(15) $u(r, \xi)=u(\xi, r)=\frac{1}{2(2-\alpha) r \xi}\left\{(r+\xi)^{2-\alpha}-|r-\xi|^{2-\alpha}\right\}$.

Hence for a positive distribution of amount $1+\nu$ spread uniformly on a spherical surface of radius $a$ and a negative one of amount $-\nu$ spread similarly on the concentric surface of radius $b, b<a$, the potential is

$$
V(\xi)=(1+\nu) u(a, \xi)-\nu u(b, \xi) .
$$

The equilibrium distribution for a given value of $b$, which we have already seen to exist, is given by the condition $V(a)=V(b)$, so that 


$$
\nu=\frac{u(b, a)-u(a, a)}{u(a, a)+u(b, b)-2 u(a, b)} .
$$

The corresponding value of $I=(1+\nu) V(a)-\nu V(b)=V(a)$ is

$$
I=u(a, a)-\nu\{u(b, a)-u(a, a)\},
$$

or

$$
I=u(a, a)-\frac{\{u(b, a)-u(a, a)\}^{2}}{u(a, a)+u(b, b)-2 u(a, b)} .
$$

We wish to show that the lower bound for $I$, as $b$ varies, is taken on for some value of $b$ in the open interval $0<b<a$. In fact, (16) and $\left(16^{\prime}\right)$ represent functions of $b$ which are continuous and differentiable except at $b=0$ and $b=a$, and it is therefore merely necessary to show that $b$ cannot approach 0 or $a$ while $I$ tends to its lower bound $I_{0}$.

Since $\alpha<1$, the potential of a positive mass distribution is strictly superharmonic. Hence $u(a, a)<u(a, \xi), \xi<a$, that is, the potential of the uniform distribution of unit positive mass on the surface of radius $a$ is greater inside than on the surface. Hence the energy can be reduced by putting a small negative mass uniformly on a sphere of radius $b, 0<b<a$, and placing the equivalent positive mass on the sphere of radius $a$. Accordingly, for the energy as given by $\left(16^{\prime}\right)$ we have $I<u(a, a)$, and therefore

$$
I_{0}<u(a, a) .
$$

But now, if we let $b$ tend to 0 in $(16)$ and $\left(16^{\prime}\right), u(b, b)$ becomes positively infinite, while the other quantities in the expressions remain finite. Hence

$$
\lim _{b=0} \nu=0 ; \quad \lim _{b=0} I=u(a, a) .
$$

Similarly, if we let $b$ tend to $a$, with $b<a$, we find by the elementary differentiation process that

$$
\lim _{b=a-0} \nu=+\infty
$$

but again, that

$$
\lim _{b=a-0} I=u(a, a)
$$


Since $u(a, a)>I_{0}$, this lower bound $I_{0}$ will be attained for some value of $b$ in the open interval $(0, a)$.

12. The Principle of the Maximum. Frostman limits his discussion in the main to functions $1 / r^{\alpha}, \alpha>1$, or to their generalization $\Phi(r)$ which retains the subharmonic property. In this case, for distributions of positive mass of total amount unity on a closed set $F$ of positive mass, the equilibrium distribution corresponding to the minimum energy is constant on $F$ except for a subset of zero capacity. This fact enables the author to extend the sweeping-out process of Poincare and Gauss. In particular there is deduced the extension of Maria's theorem, established by him for $\alpha=1,{ }^{*}$ that the upper bound of the potential of an arbitrary distribution of mass is as great on the set where the mass lies as it is on the complementary set. On account of the strictly superharmonic character of the potential when $\alpha<1$, this theorem does not hold when $\alpha<1$.

From Maria's theorem follows the further fact that, if $Q$ is a frontier point of $F, P$ a variable point of $F$, and $M$ of $C F$, then

$$
\lim _{M=Q} \sup u(M) \leqq \limsup _{P=Q} u(P),
$$

where $u(M)$ is the potential of an arbitrary distribution of positive mass on $F$. It therefore remains valid for $\alpha>1$, but even this narrower statement appears not to be true for $\alpha<1$.

The University of California

* A. J. Maria, The potential of a positive mass and the weight function of Wiener, Proceedings of the National Academy of Sciences, vol. 20 (1934), pp. 485-489. 